\title{
Just when you thought it was safe to go into the membrane: the growing complexities of extra-nuclear progesterone signaling
}

\author{
Aritro Sen and Stephen R Hammes* \\ See related research by Zuo et al., http://breast-cancer-research.com/content/12/3/R34
}

\begin{abstract}
The diversity of membrane-initiated progesterone actions has made characterization and establishment of its biological importance a complicated endeavor. A new study by Zuo and colleagues

shows that progesterone via endogenous membrane progesterone receptor-a acts as a negative regulator of proliferation and epithelial to mesenchymal transition in a breast cancer cell line. These progesterone-mediated actions appear to be regulated through epidermal growth factor receptor and phosphatidylinositol 3-kinase signaling localized in caveolae. Moreover, the study shows expression of membrane progesterone receptor-a in benign and malignant breast cancer tissues. These data bring forth novel concepts with regard to progesterone actions in the breast; however, further work is warranted to fully characterize the physiologic actions of extra-nuclear progesterone signaling in the breast.
\end{abstract}

The true role of progesterone signaling in breast cancer development and progression is not only a subject of considerable interest but is also full of controversies. The article by Zuo and colleagues [1] in this issue of Breast Cancer Research unveils a novel point of view that will further rock the field.

Classically, the perception has been that the physiological actions of progesterone are mediated via nuclear progesterone receptors (PRs) that act as transcription factors to alter gene expression [2]. However, it has long been recognized that some physiological actions of

*Correspondence: stephen_hammes@urmc.rochester.edu

Division of Endocrinology and Metabolism, Department of Medicine, University of Rochester Medical Center, Rochester, NY 14642, USA progesterone occur far too rapidly and cannot be accounted for by its genomic actions [2,3]. In the past 20 years great strides have been made in identifying various progesterone-induced rapid transcriptionindependent (non-genomic) signaling pathways. While the existence of progesterone-triggered non-genomic signaling is now relatively well accepted [2,3], the receptors mediating these actions, as well as the physiologic relevance of these extra-nuclear signals, continue to be a matter of intense debate [4,5].

Substantial evidence implicates membrane- or cytoplasmic-localized classical steroid receptors as regulators of non-genomic steroid signaling, and progesterone is no exception [3]. It has been shown that in breast cancer cells the classical PRB directly interacts with Src to mediate progesterone-triggered $\mathrm{Src}$ and downstream mitogen-activated protein kinase (MAPK) activation [6]. In fact, non-genomic PR signaling appears to promote proliferation of breast cancer cell lines under some conditions [3], although the clinical relevance of this extranuclear steroid signaling in breast cancer is unclear.

In addition to classical PRs, PGMRC1 (progesterone membrane receptor component-1) $[3,7,8]$ has been implicated in membrane-initiated progesterone signaling. PGMRC1 was first thought to be a regulator of the progesterone-induced acrosomal reaction in sperm. More recently PGMRC1 has been reported to mediate anti-apototic functions of progesterone in granulosa/ luteal cells $[3,7,8]$. However, despite these intriguing observations, stringent progesterone binding studies have not been performed, and the biological importance of PGRMC1 remains controversial. In fact, in liver microsomes, PGRMC1 has been shown to serve a completely different function as a potential cofactor for cytochrome p450 enzymes [3]. Thus, further studies are needed to establish the specificity of PGRMC1 as a PR.

Finally, in recent years, a new family of membrane PRs (the mPRs) has emerged as potential receptors for nongenomic actions of progesterone [3,7]. A vast array of 
data ranging from fish to human has shown that mPRs can modulate MAPK signaling, cAMP levels, and calcium signaling in response to progesterone $[2,3,7,9]$. Interestingly, despite having essentially no sequence homology to G protein-coupled receptors, some computer modeling programs predict mPRs to contain seven transmembrane domains [10]. Moreover, studies suggest that these receptors regulate cAMP levels via G-protein coupled signaling at the plasma membrane $[3,9]$. However, this characterization of mPRs is seriously debated by studies questioning the ability of mPRs to localize to the plasma membrane, bind to progesterone, or regulate G-protein signaling [4,5]. In addition, a recent study in yeast showed progesterone-induced signaling to be G-protein independent [11]. Thus, the functional and biological roles of the mPR family remain murky.

Despite the fair share of controversies, given the importance of progesterone actions within and outside the reproductive system, especially in the breast, mPRs remain an attractive target for therapeutic intervention and therefore a subject of intense research. The article by Zuo and colleagues [1] provides new and provocative insight into the role of mPRs in epithelial to mesenchymal transition (EMT), a differentiation process characteristic of basal phenotype breast cancer. The authors propose that progesterone acts as a negative regulator of EMT in a breast cancer cell line through $\mathrm{mPR} \alpha$-mediated mechanisms whereby $\mathrm{mPR} \alpha$ interacts with the epidermal growth factor receptor and caveolin-1 in the caveolar membrane, leading to subsequent inactivation of the phosphatidylinositol 3-kinase (PI3K)/Akt pathway and inhibition of EMT-relevant events as well as proliferation. The authors further provide evidence of $\mathrm{mPR} \alpha$ expression in benign and malignant human breast tissue, with especially high levels in triple-negative (no detectable estrogen receptor, PR, or Her2/neu) breast cancers. These observations bring forth the possibility that progestin therapy may be beneficial in triple-negative breast cancers, where their anti-EMT and anti-proliferative effects may be most potent.

To date, there is limited physiological evidence of $\mathrm{mPR}$ actions. mPRs have been implicated to have a role in the uterus $[3,7,9]$ and in the negative feedback actions of progesterone on the release of gonadotropin-releasing hormone [12]. This study by Zuo and colleagues provides the stepping stone for establishing another biological importance of mPRs in humans. However, like many scientific discoveries, the results create perhaps more questions than answers. First, the model proposed in this study indicates that the anti-EMT effects of progesterone are mediated via $\mathrm{mPR} \alpha$ by inhibition of PI3K, yet progestins are known to activate the PI3K/Akt in many other instances $[13,14]$. In fact, in the supplemental data the authors show that progesterone activates Akt phosphorylation in their cells. Second, the authors describe a negative effect of progesterone via $\mathrm{mPR} \alpha$ on MB468 cell proliferation, while others have reported a proliferative effect of PGRMC1 in the same cell type [15]. Thus, the question arises whether progesterone has both proliferative and anti-proliferative effects in breast cancer cells, depending upon the cell line and experimental setup. Third, the cells used in this study have low, but still detectable, levels of classical PR, the expression of which rises upon progesterone treatment. In addition, expression of PGRMC1 was not investigated. Therefore, it is still unclear whether $\mathrm{mPR} \alpha$ functions as a standalone PR, or whether it works together with other receptors to regulate proliferation and EMT. Finally, the true biological importance of $\mathrm{mPR}$ signaling in breast cancer development, or for that matter, in any process, cannot be properly assessed until the development of better in vivo model systems, including the creation of mPR knockout mouse models. Until then the murky waters of progesterone signaling in the membrane will remain treacherous.

\section{Abbreviations}

EMT = epithelial to mesenchymal transition; MAPK = mitogen-activated protein kinase; $\mathrm{mPR}=$ membrane progesterone receptor; $\mathrm{PGMRC1}=$ progesterone membrane receptor component-1; PI3K = phosphatidylinositol 3-kinase; $\mathrm{PR}=$ progesterone receptor.

\section{Competing interests}

The authors declare that they have no competing interests.

Published: 16 June 2010

\section{References}

1. Zuo L, Li E, You S: Progesterone reverses the mesenchymal phenotypes of basal phenotype breast cancer cells via a membrane progesterone receptor mediated pathway. Breast Cancer Res 2010, 12:R34.

2. Shupnik MA: Crosstalk between steroid receptors and the c-Src-receptor tyrosine kinase pathways: implications for cell proliferation. Oncogene 2004, 23:7979-7989.

3. Hammes SR, Levin ER: Extranuclear steroid receptors: nature and actions. Endocr Rev 2007, 28:726-741.

4. Ashley RL, Clay CM, Farmerie TA, Niswender GD, Nett TM: Cloning and characterization of an ovine intracellular seven transmembrane receptor for progesterone that mediates calcium mobilization. Endocrinology 2006, 147:4151-4159.

5. Krietsch T, Fernandes MS, Kero J, Losel R, Heyens M, Lam EW, Huhtaniemi I, Brosens JJ, Gellersen B: Human homologs of the putative $G$ proteincoupled membrane progestin receptors (mPRalpha, beta, and gamma) localize to the endoplasmic reticulum and are not activated by progesterone. Mol Endocrinol 2006, 20:3146-3164

6. Boonyaratanakornkit V, Scott MP, Ribon V, Sherman L, Anderson SM, Maller JL, Miller WT, Edwards DP: Progesterone receptor contains a proline-rich motif that directly interacts with $\mathrm{SH} 3$ domains and activates c-Src family tyrosine kinases. Mol Cell 2001, 8:269-280.

7. Zhu Y, Hanna RN, Schaaf MJ, Spaink HP, Thomas P: Candidates for membrane progestin receptors - past approaches and future challenges. Comp Biochem Physiol C Toxicol Pharmacol 2008, 148:381-389.

8. Peluso JJ: Non-genomic actions of progesterone in the normal and neoplastic mammalian ovary. Semin Reprod Med 2007, 25:198-207.

9. Zhu Y, Rice CD, Pang Y, Pace $M$, Thomas P: Cloning, expression, and characterization of a membrane progestin receptor and evidence it is an intermediary in meiotic maturation of fish oocytes. Proc Natl Acad Sci U S A 2003, 100:2231-2236 
10. Zhu Y, Bond J, Thomas P: Identification, classification, and partial characterization of genes in humans and other vertebrates homologous to a fish membrane progestin receptor. Proc Natl Acad Sci U S A 2003, 100:2237-2242.

11. Smith JL, Kupchak BR, Garitaonandia I, Hoang LK, Maina AS, Regalla LM, Lyons TJ: Heterologous expression of human mPRalpha, mPRbeta and mPRgamma in yeast confirms their ability to function as membrane progesterone receptors. Steroids 2008, 73:1160-1173.

12. Sleiter N, Pang Y, Park C, Horton TH, Dong J, Thomas P, Levine JE: Progesterone receptor A (PRA) and PRB-independent effects of progesterone on gonadotropin-releasing hormone release. Endocrinology 2009, 150:3833-3844.

13. Fu XD, Giretti MS, Baldacci C, Garibaldi S, Flamini M, Sanchez AM, Gadducci A, Genazzani AR, Simoncini T: Extra-nuclear signaling of progesterone receptor to breast cancer cell movement and invasion through the actin cytoskeleton. PLoS One 2008, 3:e2790.
14. Saitoh M, Ohmichi M, Takahashi K, Kawagoe J, Ohta T, Doshida M, Takahashi T, Igarashi H, Mori-Abe A, Du B, Tsutsumi S, Kurachi H: Medroxyprogesterone acetate induces cell proliferation through up-regulation of cyclin D1 expression via phosphatidylinositol 3-kinase/Akt/nuclear factor-kappaB cascade in human breast cancer cells. Endocrinology 2005, 146:4917-4925.

15. Ahmed IS, Rohe HJ, Twist KE, Mattingly MN, Craven RJ: Progesterone receptor membrane component 1 (Pgrmc1): a heme-1 domain protein that promotes tumorigenesis and is inhibited by a small molecule. J Pharmacol Exp Ther 2010, 333:564-573.

\section{doi: $10.1186 /$ bcr 2580}

Cite this article as: Sen A, Hammes SR: Just when you thought it was safe to go into the membrane: the growing complexities of extra-nuclear progesterone signaling. Breast Cancer Research 2010, 12:109. 